\title{
Interaction of Hsp40 with influenza virus M2 protein: implications for PKR signaling pathway
}

\author{
Zhenhong Guan ${ }^{1,2}$, Di Liư ${ }^{2}$, Shuofu $\mathrm{Mi}^{2}$, Jie Zhang ${ }^{2}$, Qinong $\mathrm{Ye}^{3}$, Ming Wang ${ }^{1}$, George F. Gao ${ }^{1,2,4}$, \\ Jinghua $\operatorname{Yan}^{2 \bowtie}$ \\ ${ }^{1}$ College of Veterinary Medicine, China Agricultural University, Beijing 100094, China \\ 2 CAS Key Laboratory of Pathogenic Microbiology and Immunology, Institute of Microbiology, Chinese Academy of Sciences, \\ Beijing 100101, China \\ 3 Beijing Institute of Biotechnology, Beijing 100850, China \\ ${ }^{4}$ Beijing Institutes of Life Science, Chinese Academy of Sciences, Beijing 100101, China \\ $\triangle$ Correspondence: yanjh@im.ac.cn
}

Received September 25, 2010 Accepted October 9, 2010

\section{ABSTRACT}

Influenza virus contains three integral membrane proteins: haemagglutinin, neuraminidase, and matrix protein (M1 and M2). Among them, M2 protein functions as an ion channel, important for virus uncoating in endosomes of virus-infected cells and essential for virus replication. In an effort to explore potential new functions of M2 in the virus life cycle, we used yeast two-hybrid system to search for M2-associated cellular proteins. One of the positive clones was identified as human $\mathrm{Hsp40/Hdj1,} \mathrm{a}$ DnaJ/Hsp40 family protein. Here, we report that both BM2 (M2 of influenza $B$ virus) and A/M2 (M2 of influenza A virus) interacted with $\mathrm{Hsp40}$ in vitro and in vivo. The region of $\mathrm{M} 2-\mathrm{Hsp40}$ interaction has been mapped to the CTD1 domain of Hsp40. Hsp40 has been reported to be a regulator of PKR signaling pathway by interacting with p58 $^{I P K}$ that is a cellular inhibitor of PKR. PKR is a crucial component of the host defense response against virus infection. We therefore attempted to understand the relationship among $M 2, H s p 40$ and $p 58^{I P K}$ by further experimentation. The results demonstrated that both $A /$ $\mathrm{M} 2$ and $\mathrm{BM} 2$ are able to bind to $\mathrm{p} 58^{\mathrm{IPK}}$ in vitro and in vivo and enhance PKR autophosphorylation probably via forming a stable complex with $\mathrm{Hsp40}$ and P58 ${ }^{\mathrm{IPK}}$, and consequently induce cell death. These results suggest that influenza virus $M 2$ protein is involved in $p 58^{I P K}$. mediated PKR regulation during influenza virus infection, therefore affecting infected-cell life cycle and virus replication.
KEYWORDS M2 protein of influenza virus, Hsp40, $\mathrm{P} 58^{\mathrm{IPK}}$, protein interaction, PKR signal pathway

\section{INTRODUCTION}

Influenza virus is an important human and zoonotic pathogen, prevalent throughout the world for centuries. The two predominant types of influenza viruses that infect humans are influenza $A$ and $B$ viruses. Both influenza $A$ and influenza $B$ viruses in the family Orthomyxoviridae have negativestranded RNA genomes consisting of eight RNA segments. RNA segments 1-3 encode the three polymerase proteins, PB1, PB2, and PA; RNA segment 4 encodes haemagglutinin (HA); RNA segment 5 encodes nucleoprotein (NP); RNA segment 6 encodes neuraminidase (NA); RNA segment 7 encodes two matrix proteins: M1 and M2; RNA segment 8 encodes two non-structural proteins NS1 and NS2 (nuclear export protein/NEP).

The M2 protein of influenza $A$ virus $(A / M 2)$ is translated from a spliced mRNA, containing 97 amino acid residues in length (Lamb et al., 1981). A/M2 has an ion channel activity to permit protons to enter virions and cause RNP-M1 dissociation during uncoating of virions in endosomes (Sugrue and Hay, 1991; Pinto et al., 1992; Takeda et al., 2002). A/M2 channel activity was required for the activation of inflammasomes by influenza and was sufficient to activate inflammasomes in primed macrophages and dendritic cells (Ichinohe et al., 2010). A/M2 also functions to equilibrate the $\mathrm{pH}$ gradient between the lumen of the trans-Golgi network (TGN) and the cytoplasm to prevent $\mathrm{HA}$ from adopting a low-pH- 
induced conformation in the Golgi apparatus (Ciampor et al., 1992; Shimbo et al., 1996). Cytoplasmic tail of the A/M2 plays a vital role in infectious virus production by coordinating the efficient packaging of genome segments into influenza virus particles (McCown and Pekosz, 2006). Unlike the A/M2 protein, the M2 protein of influenza $B$ virus (BM2) is translated from a bicistronic mRNA derived from RNA segment 7 (Briedis et al., 1982; Horvath et al., 1990), containing 109 amino acid residues, with a small $\mathrm{N}$-terminal ectodomain (1-7 aa), a single transmembrane domain (8-27 aa) and a Cterminal cytoplasmic tail (28-109 aa) (Pinto and Lamb, 2006). In addition to its obvious ion channel activity (Mould et al., 2003), BM2 protein has recently been found to be essential for influenza B virus replication as shown by the experimental data of reverse genetics technology (Hatta et al., 2004).

There is increasing evidence suggesting that the proteinprotein interactions between virus and host play an important role in the life cycle and pathogenicity of viruses (Liu et al., 2009). But so far no interacting host partners of influenza virus M2 protein have been identified, except for our recent findings of the host ATPase $\beta 1$ unit (Mi et al., 2010). In this study, a yeast two-hybrid system was used to screen a human kidney cDNA library so as to identify host proteins that interact with BM2. One clone encoding heat shock protein Hsp40 (Hdj1/ DnajB1/DjB1), a DnaJ/Hsp40 family protein, was identified. We further presented our experimental data to show that both $\mathrm{A} / \mathrm{M} 2$ and BM2 interacted with $\mathrm{Hsp} 40 / \mathrm{Hdj} 1$ in vitro and in vivo. It has been reported that $\mathrm{Hsp} 40$ associates with $\mathrm{P} 58^{\mathrm{IPK}}$, a cellular inhibitor of PKR, an interferon-induced doublestranded RNA activated serine-threonine kinase (Melville et al., 1997), which is a novel "CIHD" member of the host innate defense response against pathogenic virus. Infection of $P 58^{\mathrm{IPK}}$ knockout mice with influenza virus resulted in increased lung pathology, immune cell apoptosis, PKR activation, and mortality (Goodman et al., 2009). It has been also previously reported that Influenza virus infection promotes the disruption of the Hsp40-P58 $8^{\mathrm{IPK}}$ complex and the activation of P58 ${ }^{\mathrm{IPK}}$ (Katze et al., 1988; Lee et al., 1994; Lee and Katze, 1994). The released P58 ${ }^{\mathrm{IPK}}$ is then capable of interacting with PKR by binding to amino acids 244-296, which prevents dimerization and activation of PKR (Tan et al., 1998). Our results also show that M2 proteins could bind to $\mathrm{P} 58^{\mathrm{IPK}}$ and be able to promote PKR autophosphorylation and activation in vitro and in vivo. Therefore, it is probable that $\mathrm{M} 2$ proteins form a stable complex with Hsp40-P58 $8^{\mathrm{IPK}}$ and hinder the disassociation of Hsp40-P58 $8^{\mathrm{IPK}}$. As M2 is synthesized in the late stage of virus infection (Odagiri et al., 1999), together with the early-synthesized NS1 to inactivate the PKR activity (Bergmann et al., 2000), influenza virus regulates/ interferes cell functions to facilitate its replication through its protein products (NS1 and M2) interacting with PKR-related proteins.

\section{RESULTS}

\section{Identification of a BM2-interacting protein in the yeast two-hybrid system}

Since influenza virus is able to infect several tissues in human beings, such as lung, liver, brain etc, a prey library of human kidney cDNA was used in a yeast two-hybrid screen to identify proteins that interact with $\mathrm{BM} 2$, with the portion of the cytoplasmic domain of BM2 (BM2C) as bait. One positive clone containing cDNA with entire open reading frame encoding Hsp40/Hdj1 was isolated from a library of approximately $1 \times 10^{6}$ independent clones. To confirm the observed interaction between BM2 and Hsp40 in the yeast, we set up a growth experiment on SD plate lacking adenine, tryptophan, leucine and histidine in the two hybrid system. In this assay, growth on SD medium is supported only when the two hybrid proteins interact and induce transcription from the his reporter gene. We found that two yeast clones co-expressing $A D$ Hsp40/BD-BM2 and AD-p53/BD-T antigen (positive control) constructs grew on this medium (Fig. 1A). We also carried out liquid $\beta$-galactosidase assays by co-transforming the recombinant plasmids to yeast strain SFY526. As shown in Fig. 1B, co-transformation of AD-Hsp40 and BD-BM2C resulted in a strong induction of $\beta$-galactosidase activity, significantly higher than that of the respective control co-transformations. These results collectively indicate that BM2 and Hsp40 interact with each other.

\section{Interaction between BM2 and Hsp40 in vitro and in vivo}

To verify and extend the binding data obtained in yeast twohybrid assay, we performed GST pull-down experiments. Bacterially expressed GST-BM2C or GST bound to glutathione-Sepharose beads was allowed to react with in vitro translated $\left[{ }^{35}\right.$ S]methionine-labeled Hsp40 in reaction buffer. Consistent with the yeast two-hybrid results, Hsp40 specifically bound to GST-BM2C, but not GST (Fig. 2A).

To further assess the interaction between BM2 and Hsp40 in vivo, the plasmids that express Flag-Hsp40 or GFP-BM2 were co-transfected into 293T cells. The cell lysates were then immunoprecipitated with the anti-Flag M2-conjugated agarose and subsequently immunobloted with anti-GFP antibody. Consistent with the GST pull-down and yeast twohybrid results, BM2 specifically interacted with Hsp40 (Fig. 2B). A reciprocal co-immunoprecipitation experiment also showed the physical interaction between BM2 and Hsp40 (Fig. 2C).

\section{Mapping the BM2 binding domain of Hsp40}

To determine the region of Hsp40 that is responsible for its interaction with $\mathrm{BM} 2$, a series of $\mathrm{Hsp} 40$ deletion mutants were 
A

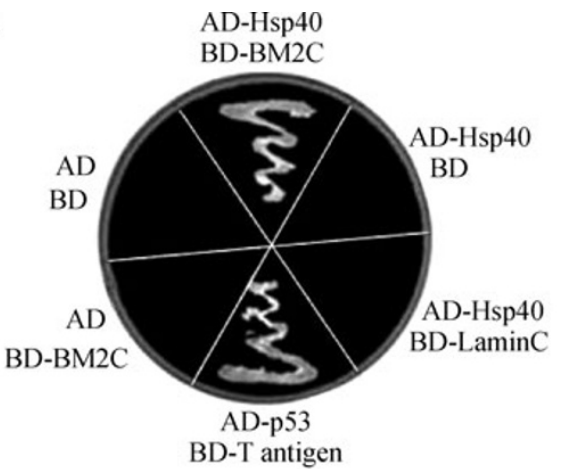

B

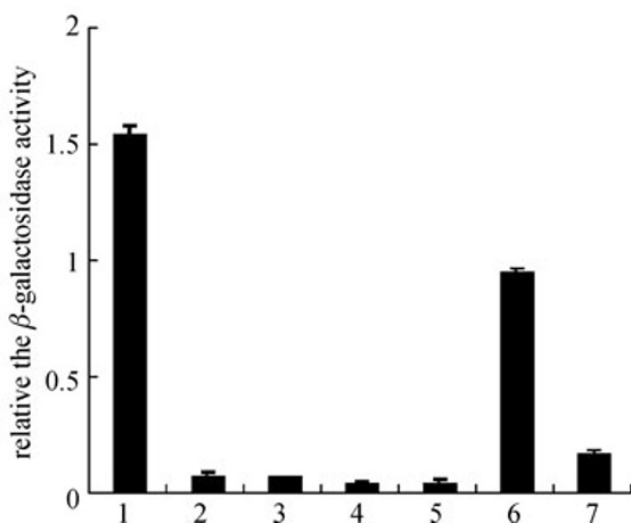

Figure 1. Yeast two-hybrid analysis. (A) Yeast strain SYF526 transformed with the indicated expression constructs were grown on an SD plate lacking adenine, tryptophan, leucine and histidine. Growth of the yeast on this plate is indication of interaction of the two expressed proteins. BD, pGBKT7 vector expressing GAL4 DNA binding domain; BD-x (e.g., BD-BM2C), the in-frame cloned plasmid of the relevant gene in pGBKT7 (pGBKT7-BM2C). AD, pACT2 vector expressing the transcription activation domain; $A D-x$ (e.g., AD-Hsp40), the in-frame cloned plasmid of the relevant gene in pACT2 (pACT2-Hsp40). BD-T antigen, BD-LaminC and ADp53 are control vectors supplied by Clontech. Interaction of BD-T antigen with AD-p53 is used as a positive control whereas BD-LaminC and AD-p53 is used as a negative control. (B) Quantification of $\beta$-galactosidase activity. Data shown are the means of three separate experiments and error bars are standard deviations. Co-transfection pair plasmid combinations are as follows: 1, pACT2-Hsp40 and pGBKT7BM2C; 2, pACT2-Hsp40 and pGBKT7; 3, pACT2-Hsp40 and pAS2-LaminC; 4, pACT2 and pGBKT7; 5, pACT2 and pGBKT7-BM2C; 6, pGBKT7-T antigen and pACT2-p53 (positive control); 7, pGBKT7-LaminC and pACT2-p53 (negative control).

constructed to test for their ability to interact with BM2 in GST pull-down assays (Fig. 3A). Full-length Hsp40 and its deletion mutants were translated, $\left[{ }^{35} \mathrm{~S}\right]-$ methionine labeled in vitro, and then incubated with GST-BM2C or GST alone. As shown in Fig. 3B, full-length Hsp40, Hsp40 (1-246 aa) including J

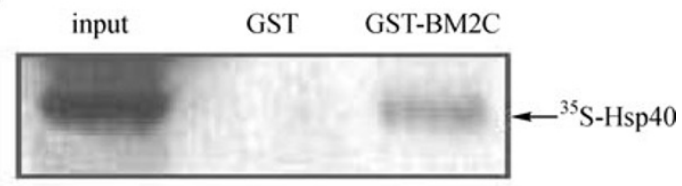

$\begin{array}{lll}\text { Flag-Hsp40 } & + & + \\ \text { GFP-BM2 } & - & + \\ \text { GFP } & + & -\end{array}$

input

IB:GFP

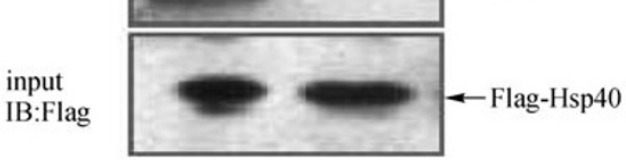

$\mathrm{C}$

$\begin{array}{lll}\text { Flag-BM2 } & + & + \\ \text { GFP-Hsp40 } & - & + \\ \text { GFP } & + & -\end{array}$

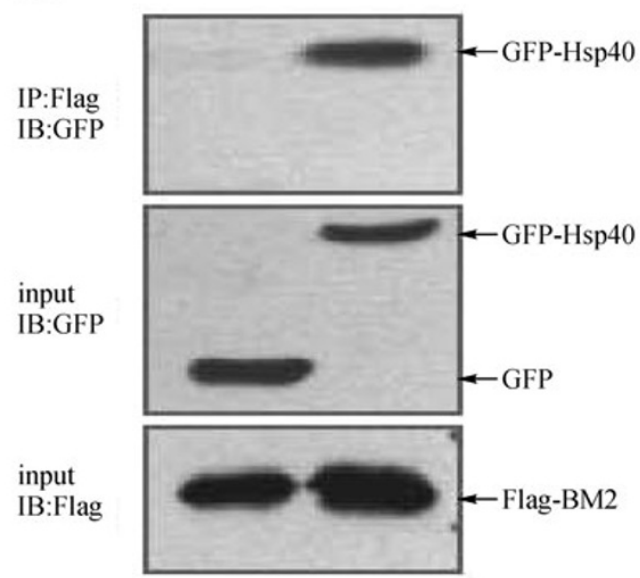

Figure 2. GST pull-down and co-immunoprecipitation (IP) experiments. (A) In vitro translated ${ }^{35} \mathrm{~S}$-labeled Hsp40 was incubated with GST or GST-BM2C fusion protein immobilized onto glutathione-Sepharose beads. Binding was viewed on SDS-PAGE by autoradiography. (B) 293T cells were cotransfected with the expression vectors for Flag-tagged Hsp40 and GFP-tagged BM2 or GFP as indicated. Lysates from the transfected cells were immunoprecipitated using antiFlag M2-agarose, and the immunoprecipitates were probed (IB) with an anti-GFP antibody (Santa Cruz). Controls of protein input and relevant IB antibodies (anti-GFP and anti-Flag) were shown in two lower panels. (C) The reciprocal co-IP assay for BM2 (flag tagged) and Hsp40 (GFP tagged) as indicated . 
A

Hsp40
Hsp40 $\triangle \mathrm{C} 2 \mathrm{D}$
Hsp40 $4 \mathrm{ClC} 2 \mathrm{D}$
Hsp40CTD1

Hsp40 $\Delta$ JG

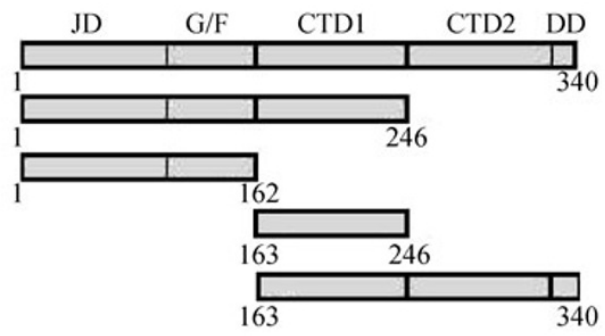

B

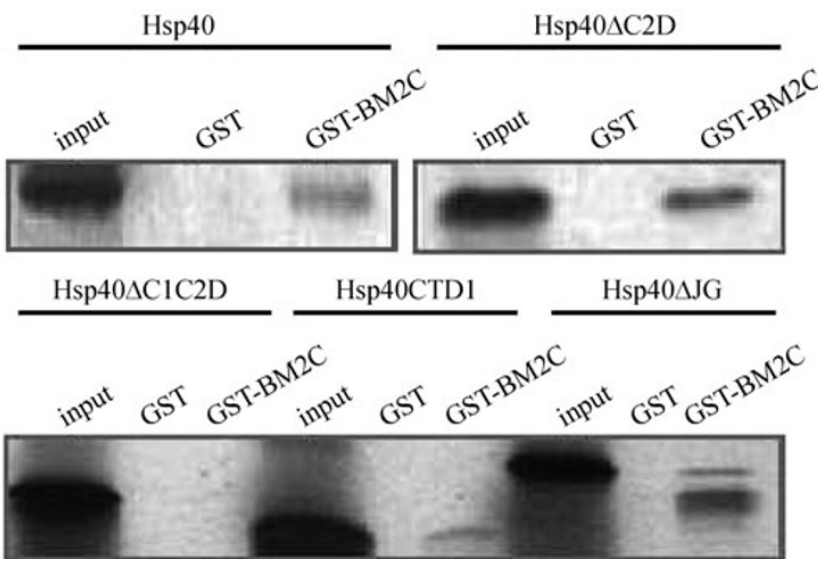

Figure 3. Definition of BM2 binding domain of Hsp40. (A) Structural representation of domain structures of wild-type Hsp40 and deletion constructs as indicated $(\Delta \mathrm{Hsp} 40)$. The subdomains of Hsp40 are labeled as follows: JD, J-domain; G/F, glycine/ phenylalanine rich domain; CTD1 and CTD2, carboxyl-terminal domain 1 and 2; DD, a predicted dimerization domain. Positions of terminal amino acids are indicated. (B) GST pull-down assays were performed using ${ }^{35}$ S-labeled Hsp40 or $\Delta \mathrm{Hsp} 40$ and GST-BM2C fusion protein. GST protein was used as a control.

domain, G/F domain and CTD1, Hsp40 (162-340 aa) including CTD1, CTD2 and DD, and Hsp40 (162-246 aa) only including CTD1 were able to interact with BM2, but not Hsp40 (1-162 aa) including $\mathrm{J}$ domain and G/F domain. As negative control, the full-length and deletion mutants of Hsp40 did not bind to GST alone. These results indicated that Hsp40 interacted with BM2 through its CTD1.

\section{Interaction between $\mathrm{A} / \mathrm{M} 2$ and $\mathrm{Hsp40}$ in vitro and in vivo}

A/M2 protein of influenza $A$ virus is structurally and biochemically similar to BM2, so we examined the possibility of A/M2 binding to Hsp40 in GST pull-down and co-IP assays. Purified GST and GST-Hsp40 immobilized on glutathioneSepharose beads were used to pull down in vitro translated and $\left[{ }^{35} \mathrm{~S}\right]$-methionine labeled $\mathrm{A} / \mathrm{M} 2$. The results indicated that Hsp40 also specifically bound to A/M2 in vitro, but not GST (Fig. 4A). To examine if $\mathrm{A} / \mathrm{M} 2$ can interact with $\mathrm{Hsp} 40$ in vivo, 293T cells were transfected with the vectors expressing FlagHsp40 or GFP-A/M2. The cell lysates were then immunoprecipitated with the anti-Flag M2-agarose and subsequently immunoblotted with anti-GFP antibody. As shown in Fig. 4B, like BM2, A/M2 also bound to Hsp40 physically in 293T cells.
A

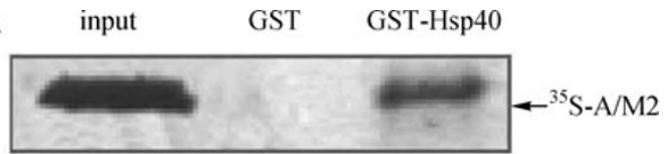

B

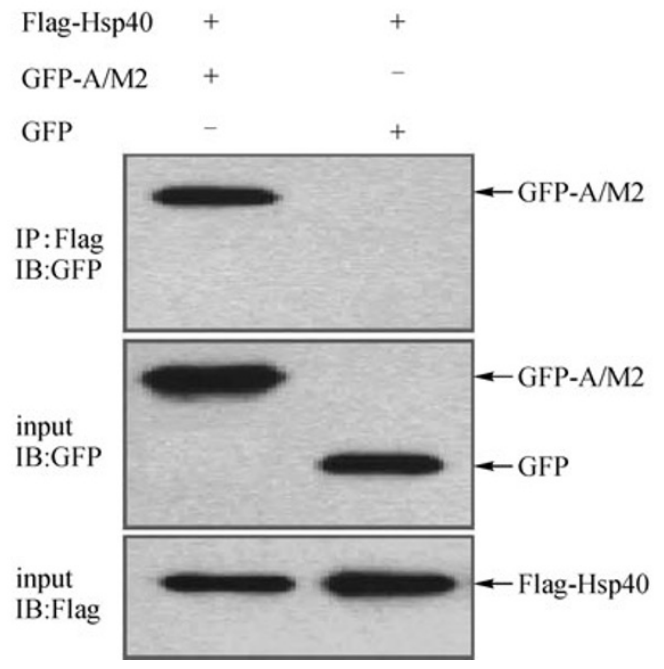

Figure 4. GST pull-down (A) and co-immunoprecipitation (IP) (B) experiments between A/M2 and Hsp40. Experiments were carried out as indicated in Fig. 2 except ${ }^{35}$ S-labeled A/M2 or the relevant $\mathrm{A} / \mathrm{M} 2$ constructs were used. 


\section{Association of M2 protein with P58 ${ }^{\mathrm{IPK}}$ and influence on the binding of Hsp40 to $\mathrm{P} 58^{\mathrm{IPK}}$ by M2 proteins}

Since it has been reported that Hsp40 interacts with $\mathrm{P} 58^{\mathrm{IPK}}$ and influenza virus infection functionally activates the $\mathrm{P} 58^{\mathrm{IPK}}$ pathway by promoting the disassociation of $\mathrm{Hsp} 40$ from $\mathrm{P} 58^{\mathrm{IPK}}$, we next investigated whether BM2 is a factor that results in their disassociation. Purified soluble GSTBM2C protein incubated with GST-P58 ${ }^{\mathrm{IPK}}$ immobilized onto glutathione-Sepharose beads, and then in vitro translated and $\left[{ }^{35} \mathrm{~S}\right]$-methionine labeled Hsp40 was added to the incubation buffer to detect the effect of BM2 on the interaction of P58 $8^{\mathrm{PK}}$ and Hsp40. Interestingly, BM2 does not block the association between $\mathrm{P} 58^{\mathrm{IPK}}$ and $\mathrm{Hsp} 40$ as we expected (Fig. 5). Using GST pull-down and co-IP approaches, we found that $\mathrm{A} / \mathrm{M} 2$ and $\mathrm{BM} 2$ were able to bind to $\mathrm{P} 58^{\mathrm{IPK}}$, In virus-infected 293T cells, overexpressed Flag-tagged P58 $8^{\mathrm{IPK}}$ was co-immunoprecipitated with M2 protein (Fig. 6). These results suggest that $\mathrm{M} 2$ protein possibly forms a stable complex with $\mathrm{Hsp} 40$ and $\mathrm{P} 58^{\mathrm{IPK}}$ and inhibits $\mathrm{P} 58^{\mathrm{IPK}}$ activity.

\section{Enhancement of PKR autophosphorylation in vitro and in vivo by M2 proteins}

$\mathrm{P} 58^{\mathrm{IPK}}$ is a negative regulator of PKR, the repression of its activity may result in increase in autophosphorylation of PKR and subsequent enhancement of phosphorylation of the $\alpha$ subunit of eukaryotic initiation factor 2 (elF2 $\alpha$ ). Therefore, to examine the functional consequences of the interaction described above, we first performed an in vitro kinase assay using purified Flag-PKR immobilized onto anti-Flag M2-conjugated agarose in the presence of GST-P58 $8^{\mathrm{IPK}}$ alone or both GST-BM2C and GST-P58 ${ }^{\mathrm{IPK}}$ as described under

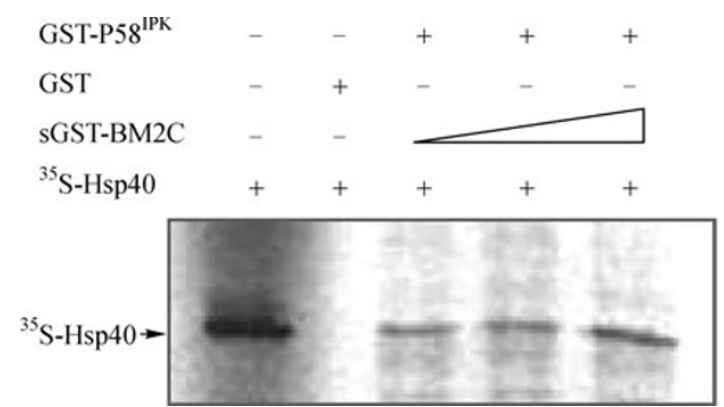

Figure 5. BM2 protein enhances binding of Hsp40 to P58 $^{\text {IPK }}$. GST-P58 ${ }^{\mathrm{IPK}}$ immobilized onto glutathione-Sepharose beads was incubated with increasing amounts of soluble GST$\mathrm{BM} 2 \mathrm{C}$ protein for $2 \mathrm{~h}$ at $4^{\circ} \mathrm{C}$, and then $\mathrm{Hsp} 40$ labeled with [ $\left.{ }^{35} \mathrm{~S}\right]-$ methionine was added to incubation buffer for $3 \mathrm{~h}$. After washing, the protein complexes were dissociated from the beads and subjected to SDS-PAGE followed by autoradiography. ${ }^{35} \mathrm{~S}-\mathrm{Hsp} 40$ signals were increasing with more GSTBM2C added. Note, left lane is ${ }^{35} \mathrm{~S}-\mathrm{H}$ sp40 input.
"Materials and Methods." As shown in Fig. 7A, the autophosphorylation of PKR was blocked by P58 ${ }^{\mathrm{IPK}}$ in vitro, and then was reversed by BM2 protein when its activator dsRNA was added.

To determine whether M2 protein has an effect on PKR autophosphorylation in mammalian cells, 293T cells were cotransfected with expression vectors for Flag-P58 $8^{\mathrm{IPK}}$ and Flag-A/M2 or Flag-BM2 constructs at 1:1 ratio and treated with IFN- $\alpha$ and poly $(\mathrm{I}: \mathrm{C})$ as described in "Materials and Methods." As a control, cells were transfected with empty vector alone. Cell extracts were then prepared and analyzed by SDS-PAGE and Western blotting with phospho-PKR antibody. As shown in Fig. 7B, overexpression of BM2 or A/ M2 in 293T cells increased the autophosphorylation of PRK due to the inhibition of P58 ${ }^{\mathrm{IPK}}$ activity via forming a complex with M2 protein.

\section{Induction of death in HeLa cells with M2 proteins}

To examine whether death was initiated in the cells transfected with pCAGGS-AM2/BM2, PI staining and flow cytometry were used to analyze the percentages of death in the total cell population. Dead cells have a weaker fluorescence (M1 zone) (Fig. 8A). Ratio of $\mathrm{M} 1$ and $\mathrm{M} 1+\mathrm{M} 2$ represents the percentages of dead cells (Fig. 8B). The results showed that HeLa cells transfected with pCAGGSAM2/BM2, exhibited cell death in a time-dependent manner (Fig. 8). The maximal cell death occurred at $72 \mathrm{~h}$ posttransfection, the dead cells increased to $13.04 \%$ and $15.78 \%$ respectively, in cells transfected with pCAGGS-AM2/BM2 In contrast, only $5.75 \%$ dead cells were observed in cells transfected with an empty vector.

\section{DISCUSSION}

Hsp40 family is involved in numerous cellular functions, including regulation of protein folding, translocation and assembly by cooperating with $\mathrm{Hsp} 70$ (Cheetham and Caplan, 1998; Ohtsuka and Hata, 2000). However, it has been recently reported that Hsp40 interacts with $\mathrm{HBV}$ core protein and inhibits viral replication (Sohn et al., 2006). Here we report for the first time that BM2 interacts with Hsp40, which has been identified by yeast two-hybrid screening and further confirmed by GST pull-down and immunoprecipitation experiments. Hsp40/Hdj1 belongs to the Type II Hsp40s, containing a J-domain, a G/F-rich domain, two conserved carboxylterminal domains (CTD1 and CTD2) and a predicted dimerization domain (Mohler et al., 2004). Our results indicated that BM2 interacted with Hsp40 through CTD1 domain. Furthermore, the data that Hsp40 was pulled down and immunoprecipitated by $\mathrm{A} / \mathrm{M} 2$ indicated that the interaction of M2 protein with Hsp40 is a common feature of influenza $A$ and $B$ viruses.

PKR is a key component in the establishment of the 
A

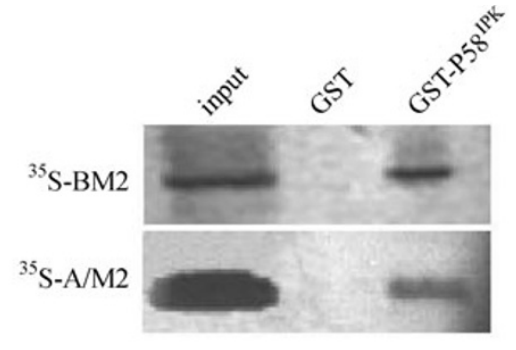

B

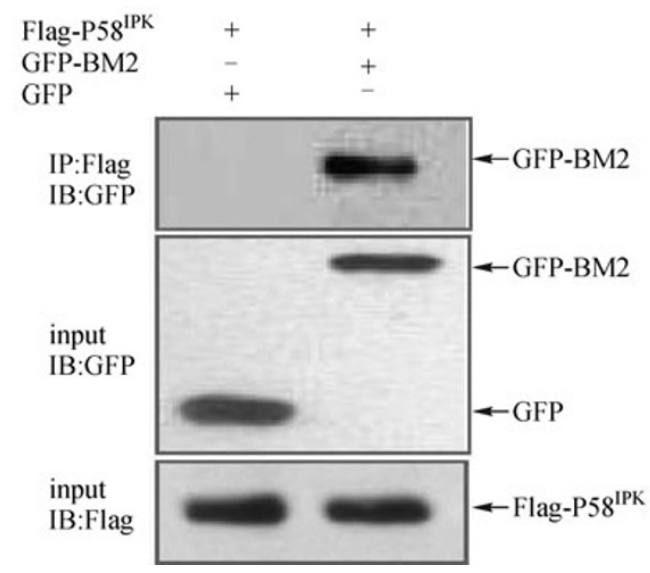

C
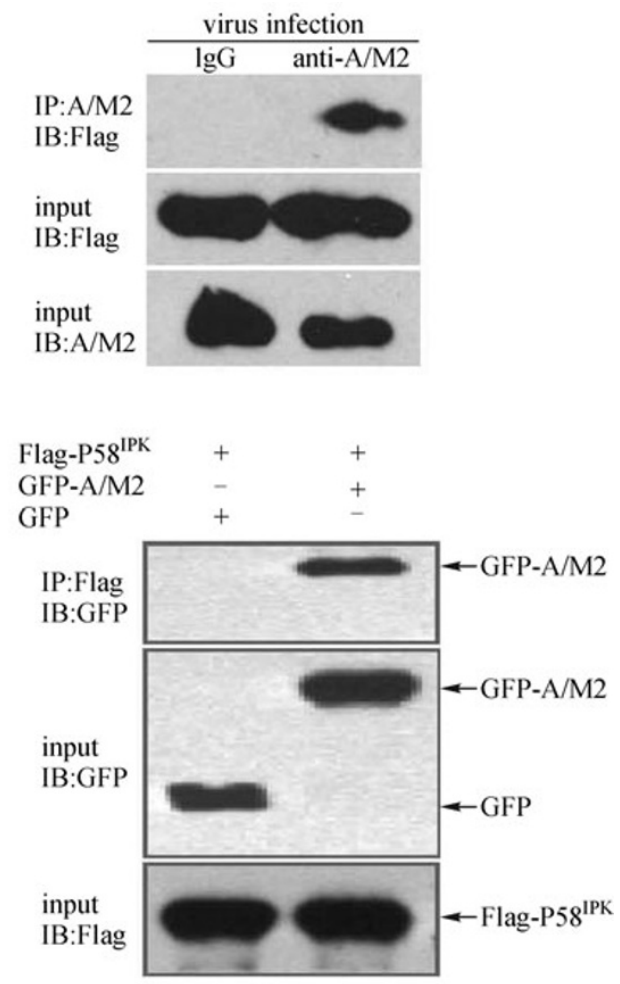

Figure 6. GST pull-down and co-immunoprecipitation (IP) to show direct interaction of P58 ${ }^{\mathrm{IPK}}$ and M2. (A) In vitro translated $\mathrm{BM} 2$ or A/M2 was incubated with GST-P58 ${ }^{\mathrm{IPK}}$ fusion protein immobilized onto glutathione-Sepharose beads. GST protein was used as a control. (B) Co-IP of P58 $8^{\mathrm{IPK}}$ and M2 protein. 293T cells growing in 6-cm-diameter dishes were transiently cotransfected with plasmids coding for $\mathrm{P} 58^{\mathrm{IPK}}$ and $\mathrm{BM} 2$ or $\mathrm{A} / \mathrm{M} 2$, and at $48 \mathrm{~h}$ after transfection, cell extracts were collected, immunoprecipitation was performed with anti-Flag antibody. and analyzed by Western blotting with GFP or Flag antibody as shown. (C) Interaction of P58 IPK and M2 protein in virus-infected cells. 293T cells transiently expressed Flag-tagged P58 ${ }^{\mathrm{IPK}}$ were infected by influenza A virus, immunoprecipitation was performed with anti-A/M2 antibody. Non-immunized mouse lgG was used as a control.

interferon-mediated cellular antiviral and antiproliferative responses (Gale et al., 1996). By binding to dsRNA, PKR undergoes a conformational change and becomes autophosphorylated at multiple serine and threonine sites, and the activated PKR then phosphorylates specific substrate elF2 $\alpha$ at Ser51, leading to an inhibition in protein synthesis and a block in viral replication (Meurs et al., 1992; Srivastava et al., 1998). Many viruses have evolved elaborate mechanisms to evade the host defense, such as production of multifunctional proteins binding to dsRNA or direct interaction with PKR (Gale and Katze, 1998). Influenza A virus has developed two strategies to block the activation of PKR. First, it encodes a non-structural protein (NS1) that can bind to dsRNA to prevent PKR autophosphorylation (Lu et al., 1995); Secondly, the infection of influenza $A$ virus activates $P 58^{\mathrm{IPK}}$, a cellular inhibitor of PKR, which can prevent dimerization and activation of PKR through directly binding to PKR. P58 $8^{\mathrm{IPK}}$ was originally characterized as an influenza virus-activated protein. Hsp40 was shown to normally bind to and negatively regulate $\mathrm{P} 58^{\mathrm{IPK}}$. The disruption of the Hsp40-P58 ${ }^{\mathrm{IPK}}$ complex was found during influenza virus infection. However, what causes the dissociation of $\mathrm{Hsp} 40$ and $\mathrm{P} 58^{\mathrm{IPK}}$ remains to be determined. Therefore, we speculate that the interaction of influenza virus M2 protein with $\mathrm{Hsp} 40$ possibly results in the dissociation of $\mathrm{Hsp} 40$ and $\mathrm{P} 58^{\mathrm{IPK}}$ and activation of P58 ${ }^{\mathrm{IPK}}$. Unexpectedly, our results from GST pull-down assay showed that purified BM2 protein could not block binding of Hsp40 to P58 ${ }^{\mathrm{PPK}}$. The further observation that both $\mathrm{BM} 2$ and $\mathrm{A} / \mathrm{M} 2$ can bind to $\mathrm{P} 58^{\mathrm{IPK}}$ suggests that $\mathrm{M} 2$ protein, $\mathrm{Hsp} 40$ and $\mathrm{P} 58^{\mathrm{IPK}}$ probably form a stable complex in virus-infected cells. The findings that the level of PKR autophosphorylation was enhanced by both $A / M 2$ and $B M 2$ in vitro and in vivo indicated that $\mathrm{P} 58^{\mathrm{IPK}}$ was arrested and inactivated as a result of interaction with $\mathrm{Hsp} 40$ and $\mathrm{M} 2$ protein.

Studies using the herpes simplex virus translocating protein VP22 to carry influenza virus proteins into cells have shown that expression of M2 protein induces Hela cell apoptosis (Morris et al., 2002). It is reported that $A / M 2$ is highly toxic for mammalian cells, yeast and insect cells (llyinskii et al., 2007, 2008). Similar observations have been made in our experiments. Taken together, our results demonstrate that, during influenza virus infection, M2 protein 

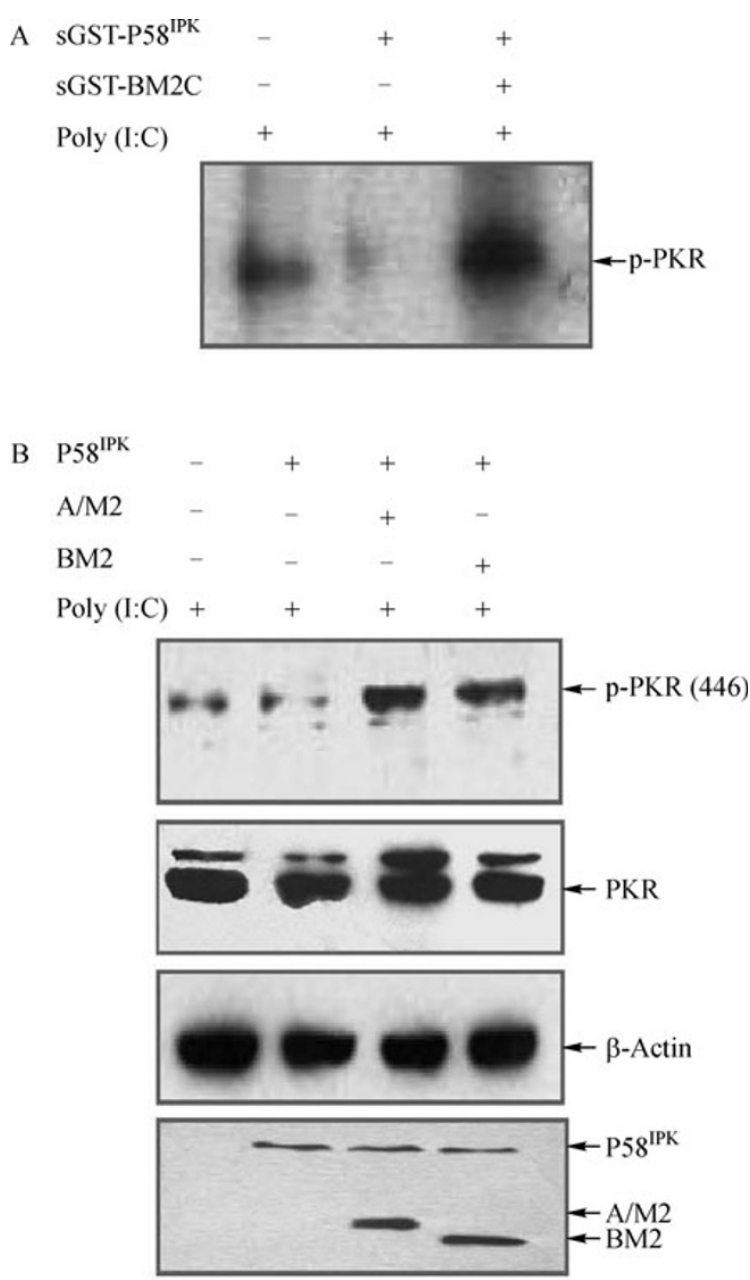

Figure 7. $M 2$ (BM2 and $A / M 2$ respectively) protein enhances PKR phosphorylation. (A) Flag-PRK immobilized onto anti-Flag M2-conjugated agarose was incubated with purified soluble GST control (lane 1), GST-P58 ${ }^{\mathrm{IPK}}$ (lane 2) and the complex of GST-BM2C and GST-P58 ${ }^{\text {IPK }}$ (lane 3) in the presence of $\left[{ }^{32} \mathrm{P}\right]$ ATP. Reaction mixtures were subjected to SDS-PAGE and visualized by autoradiography. (B) Overexpression of M2 enhances PRK phosphorylation. 293T cells were transfected with the indicated plasmids encoding for FlagP58 ${ }^{\mathrm{IPK}}$, Flag-A/M2, or Flag-BM2 and treated with IFN- $\alpha$ and poly $(\mathrm{I}: \mathrm{C})$ as described in "Materials and Methods." Equal amounts of cell extracts were analyzed by SDS-PAGE and subjected to immunoblotting with phospho-PKR, PKR and $\beta$ actin antibody. The top two panels show protein levels of phosphorylated PKR and total PKR detected by phospho-PKR (P-PKR) and PKR antibodies (PKR). The third panel shows protein level of $\beta$-actin acted as a loading control. The bottom panel shows protein levels of $\mathrm{P} 58^{\mathrm{IPK}}, \mathrm{A} / \mathrm{M} 2$ and $\mathrm{BM} 2$ detected by Western blotting.

associating with host partners $\mathrm{Hsp} 40$ and $\mathrm{P} 58^{\mathrm{IPK}}$ leads to the autophosphorylation of PKR, reduction of host proteins synthesis, and finally, induction of cell apoptosis.
It has been thought that the induction of apoptosis is a host defense response, stopping the replication and spread of virus. However, the increasing evidence has shown that apoptosis induction is beneficial for influenza virus replication. First of all, the expression of anti-apoptosis protein Bcl-2 which inhibits influenza virus-induced apoptosis reduces virus replication, spread and HA glycosylation (Olsen et al., 1996). Furthermore, the inhibition of caspase 3 activity which is a member of the central component of the apoptotic machinery strongly impairs influenza virus propagation (Wurzer et al., 2003). It seems that influenza virus has acquired the capability to take advantage of the protection machinery of the host cells, thereby supporting viral replication. The virus probably needs some mechanisms to keep the balance between limitation of antiviral response and maintenance of sufficient signaling strength to support virus growth. Such a balance may be controlled by proteins encoded by influenza virus. There are several proteins of influenza virus that has been reported to act as apoptosis promoters: NS1, PB1-F2, NA and M2. But the data that NS1 acts as apoptosis inducer (Schultz-Cherry et al., 2001) was challenged by the finding that recombinant influenza virus lacking NS1 still induced cell apoptosis. It is expressed during the early stage of infection and has been described as an inhibitor of PKR to promote viral protein synthesis. NA was the first influenza virus protein shown to have a role in the induction of apoptosis (Schultz-Cherry and Hinshaw, 1996; Morris et al., 1999). It can activate TGF- $\beta$ at the cell surface by facilitating cleavage of TGF- $\beta$ into its active form. However, NA is not the sole contributor to apoptosis as UV-irradiated virus, which retains $100 \%$ NA activity, weakly induced apoptosis. PB1-F2 is known to localize in the mitochondria of the infected cell and to sensitize cells to death through interactions with two mitochondrial proteins, ANT3 and VDAC1 (Zamarin et al., 2005). These interactions promote the permeabilization of the mitochondria, facilitate the release of mitochondrial products and trigger cell apoptosis. Like PB1-F2, M2 protein is expressed during the later stages of the infection cycle. This correlates well with late requirement for TRAIL and caspase activity in the viral replication cycle. Their pro-apoptotic effect most likely is not inhibitory to viral replication.

Based on our results, we propose the following model for the regulation of PKR pathway by influenza virus proteins during the infection (Fig. 9). PKR remains latent in unstimulated cells, and its activation requires binding of specific activators. $\mathrm{P} 58^{\mathrm{IPK}}$ is also inactive before influenza virus infection because it is bound to Hsp40. Early in the infection, PKR is activated by dsRNA generated by viruses. Moreover, NS1 is expressed and blocks the dsRNA-mediated activation of PKR to fight against host defense and support normal viral replication. $\mathrm{P} 58^{\mathrm{IPK}}$ is also activated to block the dimerization and activation of PKR due to the disruption of Hsp40-P58 $8^{\text {IPK }}$ complex. During later stage of the infection, M2 is expressed 
A
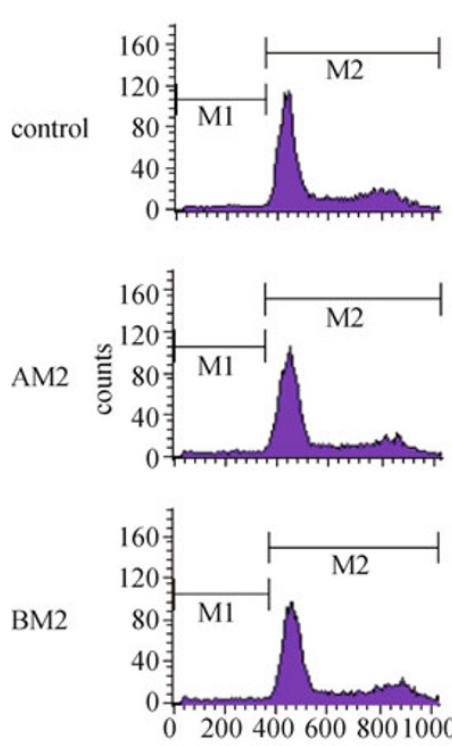

B
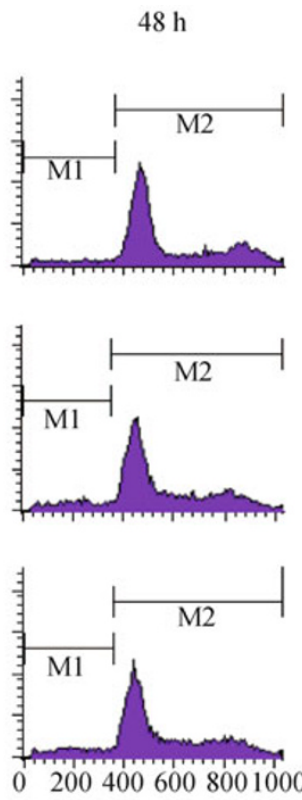

FL2-A
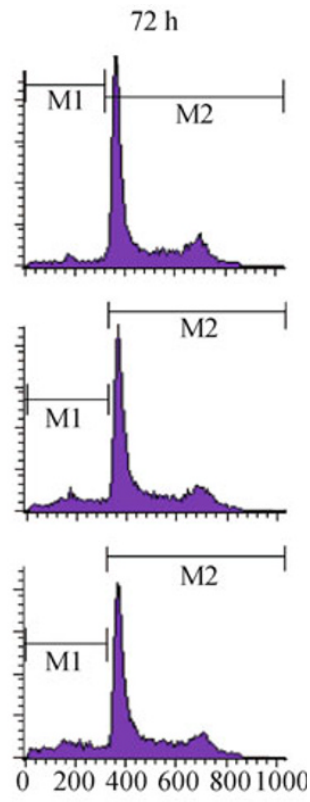

02004006008001000

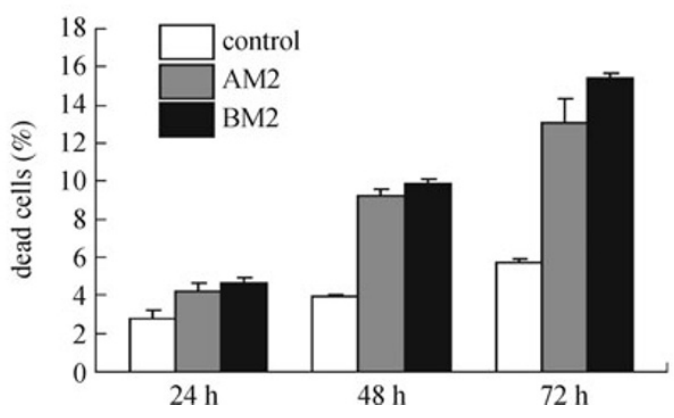

Figure 8. Supravital PI assays identify the dead cells. HeLa cells were transfected with plasmid pCAGGS-AM2/BM2, stained with PI (see Methods) and analyzed by flow cytometry. (A) Representative DNA histograms showing the proportions of dead hypodiploid nuclei detected by flow cytometry at 24-72 h. (B) Proportions of dead cells by PI staining after transfected with pCAGGS, pCAGGS-AM2 or pCAGGS-BM2 at 24-72 h. Results are expressed as means \pm SD of results from three independent experiments. Control, cells transfected with pCAGGS empty vector; AM2, cells transfected with pCAGGS-AM2; BM2, cells transfected with pCAGGS-BM2.

and associates with $\mathrm{Hsp} 40$ and $\mathrm{P} 58^{\mathrm{IPK}}$ to prevent the disruption of Hsp40-P58 $8^{\mathrm{IPK}}$ complex. This leads to the activation of PKR, and then may induce cell apoptosis and control virus replication. Through this sophisticated way, influenza virus manipulates the host cells to favor its replication and release.

\section{METHODS}

\section{Plasmid construction}

To generate GST or Flag epitope-tagged full-length protein of Hsp40, PCR was performed to amplify Hsp40 DNA fragment from the original yeast two-hybrid library clone (pACT2Hsp40) (see below). The PCR product was digested with
BamH I-Xho I and then inserted into pGEX6p-1 and pcDNA3Flag (Clontech). Deletion mutants of Hsp40 were constructed by inserting PCR-generated fragments from the corresponding cDNAs into the pcDNA3-Flag vector. The mammalian expression plasmids of wild-type BM2 and A/M2 fused with Flag epitope were generated by constructing to pCAGGS/ MCS vector (kindly provided by $\mathrm{Dr} \mathrm{Y}$. Kawaoka, The University of Tokyo). The plasmid pcDNA3-Flag-PKR was constructed to use for purification of PKR protein by amplifying a full-length PKR cDNA from a human kidney cDNA library (Clontech) and then inserting to pcDNA3-Flag vector. For the expression of $\mathrm{P} 58^{\mathrm{PK}}$ in mammalian cells and E. coli, a P58 ${ }^{\mathrm{IPK}}$ CDNA was obtained from Hela cells by RT$P C R$ and inserted to pcDNA3-Flag and pGEX6p-1 vector, respectively. The PCR fragment product (BM2C) of cytoplas- 


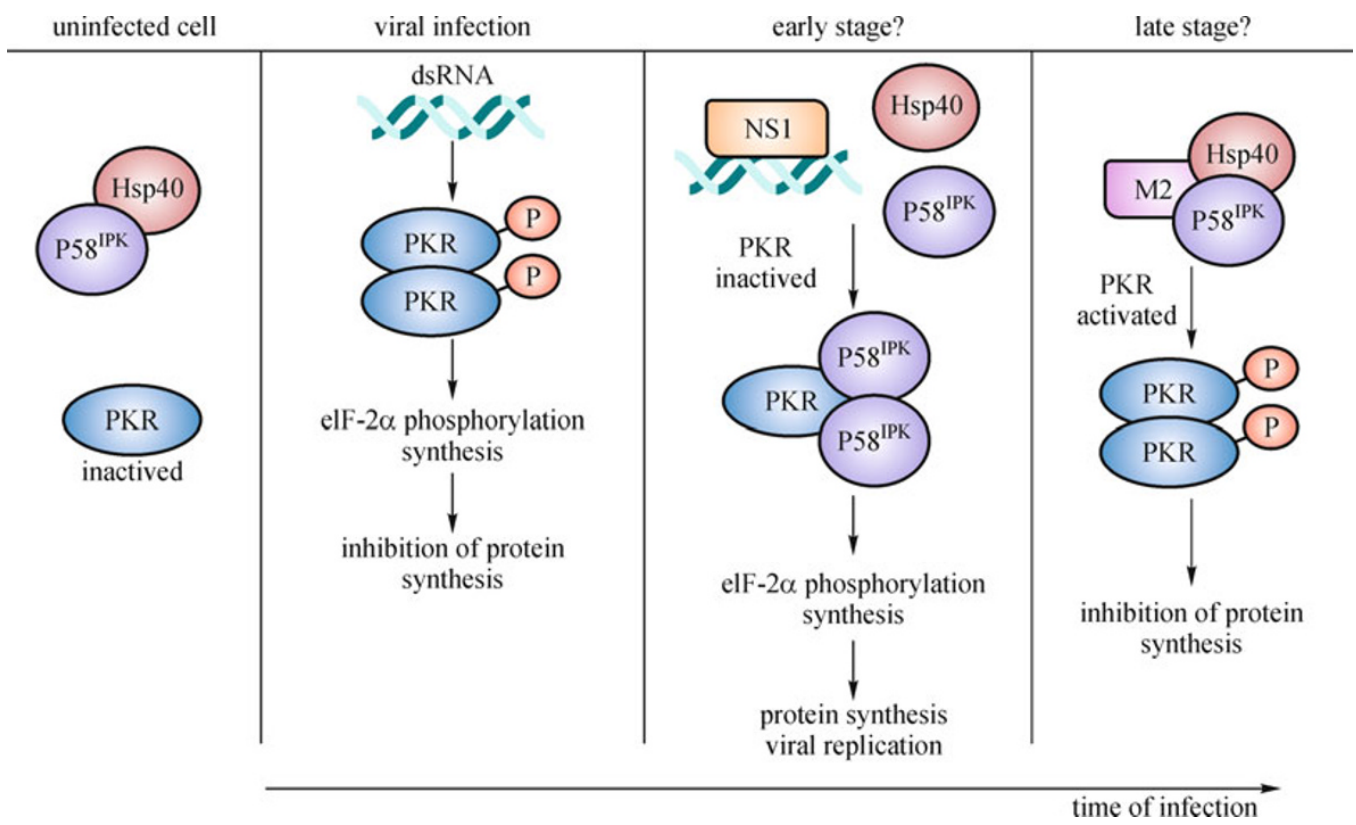

Figure 9. Proposed model of modulation of PKR function by influenza virus. In the uninfected cells, both $P K R$ and P58 $8^{I P K}$ are in inactivated state. At the start of virus infection, viral dsRNA activates PKR but in the early stage of influenza virus infection, the early-synthesized protein NS1 binds to dsRNA thereof promoting the viral protein synthesis. In the late stage, late-synthesized viral M2 activates PKR through its interaction with $\mathrm{Hsp} 40$ and P58 $8^{\mathrm{IPK}}$ to inhibit host protein synthesis therefore promoting the virus packaging and budding (for detail, please see text).

mic domain (amino acids 28-109) of the BM2 gene derived from Influenza $B$ virus strain (B/Yamagata/K542/2001) was inserted into pGBKT7 (Clontech) in frame with GAL4 DNA binding domain (BD), resulting into plasmid pGBKT7BM2C.

\section{Yeast two-hybrid screening}

For the initial screening, pGBKT7-BM2C was used as bait and PACT cDNA library (Clontech) from human kidney was used as a source of prey genes. The bait pGBKT7-BM2C plasmid and the pACT2 cDNA library were transformed into the yeast strain $\mathrm{AH} 109$ by lithium acetate method (yeast protocols handbook, Clontech). Transformants were plated onto SD medium lacking tryptophan, leucine and histidine but containing $1 \mathrm{mM} 3$-aminotriazole. The candidate clones were rescued from the yeast cells and introduced to the yeast strain SFY526 to verify the interaction by detecting $\beta$-galactosidase activity. For quantitative $\beta$-galactosidase assays, colonies were grown to mid-logarithmic phase in liquid selection medium before cells were harvested and lysed by the glass-bead method (yeast protocols handbook, Clontech). The procedures for library amplification, yeast cell transformation, screening for growth in the absence of histidine, and measurement of $\beta$-galactosidase activity followed the Matchmaker protocol (Clontech).

\section{Cell lines and reagents}

293T and Hela cells were cultured in high-glucose DMEM (Gibco) supplemented with $10 \%$ heat-inactivated fetal bovine serum (FBS), $2 \mathrm{mM}$ L-glutamine, and penicillin-streptomycin (100 units/mL; Invitrogen). Anti-Flag monoclonal M2 antibody, M2 anti-Flag-agarose, and poly(l:C) (synthetic dsRNA) were purchased from Sigma. The polyclonal anti-PKR antibody ( $N$ 18; sc-6282) and phospho-specific anti-PKR antibody (against phosphorylated threonine 446) were purchased from Santa Cruz Biotechnology.

\section{Cell lysis and immunoblotting experiments}

To analyze whether M2 protein affects PKR autophosphorylation in vivo, we performed the following assays. 293T cells were transfected with Flag-tagged A/M2, BM2 and/or P58 ${ }^{\mathrm{IPK}}$ constructs. Twenty-four hours later, the cells were treated with alpha interferon (IFN- $\alpha$ ) at $1000 \mathrm{U} / \mathrm{mL}$ for $24 \mathrm{~h}$, and then poly (I:C) $(100 \mu \mathrm{g} / \mathrm{mL})$ was added directly to the cell culture medium for $6 \mathrm{~h}$ to activate PKR. To harvest, the cells were washed once with PBS buffer and proteins were extracted in ice-cold lysis buffer containing $50 \mathrm{mM}$ Tris- $\mathrm{HCl}(\mathrm{pH} 8.0)$, $150 \mathrm{mM} \mathrm{NaCl}, 0.5 \% \mathrm{NP}-40,1 \mathrm{mM}$ dithiothreitol (DTT), $1 \mathrm{mM}$ phenylmethylsulfonyl fluoride (PMSF), CPI cocktail (Boehringer Mannheim) as the source of protease inhibitors. The 
extracts were clarified by microcentrifugation at $13,000 \mathrm{rpm}$ for $10 \mathrm{~min}$. The protein concentration was determined by bicinchoninic acid (BCA) protein assay kit (Pierce Biotechnology), and $40 \mu \mathrm{g}$ of protein were fractionated by sodium dodecyl sulfate-polyacrylamide gel electrophoresis (SDSPAGE). The proteins were then electroblotted onto PVDF membrane, which was incubated with each of the following antibodies: phosphorylation site-specific antibody to PKR (Thr446), rabbit polyclonal antibodies to PKR and to $\beta$-actin as a control for protein loading. The secondary antibodies were horseradish peroxidase-conjugated anti-rabbit or antigoat IgG (Amersham Biosciences) used at a dilution of 1:5000 in blocking solution.

\section{Co-immunoprecipitation (Co-IP) assay}

Immunoprecipitation assays were performed essentially as described previously (Yan et al., 2003; Fan et al., 2006). Briefly, 293T cells were transiently transfected with the indicated plasmids using Lipofectamine 2000 reagents (Invitrogen). After $48 \mathrm{~h}$ of cultivation, the cells were washed and resuspended in $0.5 \mathrm{~mL}$ lysis buffer (mentioned above). Equal amounts of cleared cell lysates were subjected to immunoprecipitate with anti-Flag monoclonal antibody M2conjugated agarose. The reactions were performed overnight at $4^{\circ} \mathrm{C}$, and then the beads were centrifuged at $3000 \mathrm{rpm}$ for $2 \mathrm{~min}$ and washed three times with lysis buffer. The antibodyprotein complexes were then resolved by SDS-PAGE, and the GFP or Flag-tagged proteins were identified by Western blotting with an anti-GFP/Flag antibody probe using an enhanced chemiluminescence system. For the detection of interaction between $\mathrm{P} 58^{\mathrm{IPK}}$ and $\mathrm{A} / \mathrm{M} 2$ in the virus-infected cells, 293T cells were transfected with pFlag- P58 $8^{\mathrm{IPK}}$ plasmid for $24 \mathrm{~h}$, and then infected by influenza virus A/WSN/33. After $24 \mathrm{~h}$, co-immunoprecipitation was performed with anti-A/M2 antibody (Abcam), non-immunized mouse IgG as a negative control.

\section{Preparations of GST fusion proteins and GST pull-down assay}

The interactions between $\mathrm{M} 2$ and $\mathrm{Hsp} 40$ or $\mathrm{P} 58^{\mathrm{IPK}}$ in vitro were examined by GST pull-down assays. Transformants of E. coli BL21 bearing plasmids encoding GST or different GST fusion proteins were grown to an optical density at $600 \mathrm{~nm}$ $\left(\mathrm{OD}_{600}\right)$ of $0.6-0.7$, and IPTG was added to $0.1 \mathrm{mM}$ to induce expression of the GST proteins for $12 \mathrm{~h}$ at $16^{\circ} \mathrm{C}$. Total proteins were extracted by sonicating cells in PBS buffer containing NP-40 and protein inhibitors, followed by centrifugation at $12,000 \times \mathrm{g}$ for $15 \mathrm{~min}$ at $4^{\circ} \mathrm{C}$. The supernatant were purified by glutathione-Sepharose $4 \mathrm{~B}$ beads according to the manufacturer's recommended protocol (Amersham Pharmacia Biotech).

${ }^{35}$ S-labeled A/M2/BM2, Hsp40 and P58 ${ }^{\mathrm{IPK}}$ proteins were produced by in vitro transcription and translation using plasmids pcDNA3/BM2, pcDNA3/Hsp40, pcDNA3/P58 ${ }^{\mathrm{IPK}}$, respectively. $\left[{ }^{35} \mathrm{~S}\right]$-Pro Mix (mixture of $\left[{ }^{35}\right.$ S]methionine; Amersham) and the TNT T7 coupled reticulocyte lysate system (Promega) were used as instructed by the manufacturer. GST binding assays were conducted as follows: The ${ }^{35}$ S-labeled proteins were incubated with $10 \mu \mathrm{g}$ of GST derivatives bound to glutathione-Sepharose beads in $0.5 \mathrm{~mL}$ binding buffer ( $50 \mathrm{mM}$ Tris- $\mathrm{HCl}(\mathrm{pH} 8.0), 150 \mathrm{mM} \mathrm{NaCl}, 1 \mathrm{mM}$ EDTA, $0.5 \%$ NP-40, $1 \mathrm{mM}$ DTT, $1 \mathrm{mM}$ PMSF and protease inhibitors). The binding reaction was performed at $4^{\circ} \mathrm{C}$ overnight and the beads were subsequently washed four times with the binding buffer. The beads were then resuspended in $10 \mu \mathrm{L}$ of $2 \times$ SDS sample buffer, resolved by SDS-PAGE and followed by autoradiography.

\section{Protein kinase assay}

Purified recombinant Flag-tagged PKR immunoprecipitated from cell extracts were used for the in vitro kinase reactions in kinase reaction buffer containing $20 \mathrm{mM}$ HEPES, $\mathrm{pH} 7.4$, $1 \mathrm{mM}$ DTT, $5 \mathrm{mM} \mathrm{MgCl}_{2}, 20 \mu \mathrm{M}$ ATP, $5 \mu \mathrm{Ci}$ of $\left[\gamma^{-32}\right.$ P]ATP $(6 \mathrm{mCi} / \mathrm{mmol})$. Whenever indicated, poly(l:C) was added to a final concentration of $1 \mu \mathrm{g} / \mathrm{mL}$. The kinase reactions were incubated for $30 \mathrm{~min}$ at $30^{\circ} \mathrm{C}$, stopped by the addition of $2 \times$ SDS-PAGE sample buffer, boiled for $5 \mathrm{~min}$, and analyzed by SDS-PAGE (10\%). The degree of PKR phosphorylation was visualized by autoradiography.

\section{Cell death assay}

HeLa cells were cultured in 6-well plates and allowed to grow to $75 \%-80 \%$ confluency, and then were transfected with pCAGGS-AM2/BM2 ( $3 \mu \mathrm{g} / \mathrm{well})$. Cells were collected at 24,48 and $72 \mathrm{~h}$ post-transfection, washed twice with PBS and fixed by $75 \%$ cooling ethanol overnight. The fixed cells were then stained with $50 \mu \mathrm{g} / \mathrm{mL} \mathrm{PI}$ in the dark at room temperature for $15 \mathrm{~min}$. A minimum of $1 \times 10^{5}$ cells for each group was analyzed by fluorescence activated cell sorting (FACS).

\section{ACKNOWLEDGEMENTS}

This work was supported by National Natural Sciences Foundation of China (NSFC) (Grant Nos. 30670091 and 30599434), National Basic Research Program (Project 973) of China Ministry of Science and Technology (Grant No. 2011CB504703), National Key Technologies R\&D Program (Grant No. 2006BAD06A01). GFG is a leading principal investigator of the NSFC Innovative Research Group (Grant No. 81021003).

\section{REFERENCES}

Bergmann, M., Garcia-Sastre, A., Carnero, E., Pehamberger, H., Wolff, K., Palese, P., and Muster, T. (2000). Influenza virus NS1 protein counteracts PKR-mediated inhibition of replication. J Virol $74,6203-6206$. 
Briedis, D.J., Lamb, R.A., and Choppin, P.W. (1982). Sequence of RNA segment 7 of the influenza $B$ virus genome: partial amino acid homology between the membrane proteins (M1) of influenza $A$ and $B$ viruses and conservation of a second open reading frame. Virology 116, 581-588.

Cheetham, M.E., and Caplan, A.J. (1998). Structure, function and evolution of DnaJ: conservation and adaptation of chaperone function. Cell Stress Chaperones 3, 28-36.

Ciampor, F., Thompson, C.A., Grambas, S., and Hay, A.J. (1992). Regulation of $\mathrm{pH}$ by the $\mathrm{M} 2$ protein of influenza $A$ viruses. Virus Res 22, 247-258.

Fan, Z., Zhuo, Y., Tan, X., Zhou, Z., Yuan, J., Qiang, B., Yan, J., Peng, X., and Gao, G.F. (2006). SARS-CoV nucleocapsid protein binds to hUbc9, a ubiquitin conjugating enzyme of the sumoylation system. J Med Virol 78, 1365-1373.

Gale, M. Jr, and Katze, M.G. (1998). Molecular mechanisms of interferon resistance mediated by viral-directed inhibition of PKR, the interferon-induced protein kinase. Pharmacol Ther 78, 2946.

Gale, M. Jr, Tan, S.L., Wambach, M., and Katze, M.G. (1996). Interaction of the interferon-induced PKR protein kinase with inhibitory proteins P58IPK and vaccinia virus $\mathrm{K} 3 \mathrm{~L}$ is mediated by unique domains: implications for kinase regulation. Mol Cell Biol 16, 4172-4181.

Goodman, A.G., Fornek, J.L., Medigeshi, G.R., Perrone, L.A., Peng, X., Dyer, M.D., Proll, S.C., Knoblaugh, S.E., Carter, V.S., Korth, M. J., et al. (2009). P58(IPK): a novel "CIHD" member of the host innate defense response against pathogenic virus infection. PLoS Pathog 5, e1000438.

Hatta, M., Goto, H., and Kawaoka, Y. (2004). Influenza B virus requires BM2 protein for replication. J Virol 78, 5576-5583.

Horvath, C.M., Williams, M.A., and Lamb, R.A. (1990). Eukaryotic coupled translation of tandem cistrons: identification of the influenza B virus BM2 polypeptide. EMBO J 9, 2639-2647.

Ichinohe, T., Pang, I.K., and Iwasaki, A. (2010). Influenza virus activates inflammasomes via its intracellular M2 ion channel. Nat Immunol 11, 404-410.

Ilyinskii, P.O., Gabai, V.L., Sunyaev, S.R., Thoidis, G., and Shneider, A.M. (2007). Toxicity of influenza A virus matrix protein 2 for mammalian cells is associated with its intrinsic proton-channeling activity. Cell Cycle 6, 2043-2047.

Ilyinskii, P.O., Gambaryan, A.S., Meriin, A.B., Gabai, V., Kartashov, A., Thoidis, G., Shneider, A.M., and Blagosklonny, M. (2008). Inhibition of influenza M2-induced cell death alleviates its negative contribution to vaccination efficiency. PLoS ONE 3, e1417.

Katze, M.G., Tomita, J., Black, T., Krug, R.M., Safer, B., and Hovanessian, A. (1988). Influenza virus regulates protein synthesis during infection by repressing autophosphorylation and activity of the cellular $68,000-\mathrm{Mr}$ protein kinase. J Virol 62, 3710-3717.

Lamb, R.A., Lai, C.J., and Choppin, P.W. (1981). Sequences of mRNAs derived from genome RNA segment 7 of influenza virus: colinear and interrupted mRNAs code for overlapping proteins. Proc Natl Acad Sci U S A 78, 4170-4174.

Lee, S.B., Green, S.R., Mathews, M.B., and Esteban, M. (1994). Activation of the double-stranded RNA (dsRNA)-activated human protein kinase in vivo in the absence of its dsRNA binding domain. Proc Natl Acad Sci U S A 91, 10551-10555.
Lee, T.G., and Katze, M.G. (1994). Cellular inhibitors of the interferoninduced, dsRNA-activated protein kinase. Prog Mol Subcell Biol $14,48-65$.

Liu, D., Liu, X., Yan, J., Liu, W.J., and Gao, G.F. (2009). Interspecies transmission and host restriction of avian $\mathrm{H} 5 \mathrm{~N} 1$ influenza virus. Sci China C Life Sci 52, 428-438.

Lu, Y., Wambach, M., Katze, M.G., and Krug, R.M. (1995). Binding of the influenza virus NS1 protein to double-stranded RNA inhibits the activation of the protein kinase that phosphorylates the elF-2 translation initiation factor. Virology 214, 222-228.

McCown, M.F., and Pekosz, A. (2006). Distinct domains of the influenza a virus M2 protein cytoplasmic tail mediate binding to the M1 protein and facilitate infectious virus production. J Virol 80, 8178-8189.

Melville, M.W., Hansen, W.J., Freeman, B.C., Welch, W.J., and Katze, M.G. (1997). The molecular chaperone hsp40 regulates the activity of P58IPK, the cellular inhibitor of PKR. Proc Natl Acad Sci U S A 94, 97-102.

Meurs, E.F., Watanabe, Y., Kadereit, S., Barber, G.N., Katze, M.G., Chong, K., Williams, B.R., and Hovanessian, A.G. (1992). Constitutive expression of human double-stranded RNA-activated p68 kinase in murine cells mediates phosphorylation of eukaryotic initiation factor 2 and partial resistance to encephalomyocarditis virus growth. J Virol 66, 5805-5814.

Mi, S., Li, Y., Yan J., and GAO, G.F. (2010). Na $/ K^{+}-A T P a s e ~ \beta 1$ subunit interacts with $M 2$ proteins of influenza $A$ and $B$ viruses and affects the virus replication. Sci China C Life Sci 53, 1-8.

Mohler, P.J., Hoffman, J.A., Davis, J.Q., Abdi, K.M., Kim, C.R., Jones, S.K., Davis, L.H., Roberts, K.F., and Bennett, V. (2004). Isoform specificity among ankyrins. An amphipathic alpha-helix in the divergent regulatory domain of ankyrin-b interacts with the molecular co-chaperone Hdj1/Hsp40. J Biol Chem 279, 25798-25804.

Morris, S.J., Price, G.E., Barnett, J.M., Hiscox, S.A., Smith, H., and Sweet, C. (1999). Role of neuraminidase in influenza virus-induced apoptosis. J Gen Virol 80, 137-146.

Morris, S.J., Smith, H., and Sweet, C. (2002). Exploitation of the Herpes simplex virus translocating protein VP22 to carry influenza virus proteins into cells for studies of apoptosis: direct confirmation that neuraminidase induces apoptosis and indications that other proteins may have a role. Arch Virol 147, 961-979.

Mould, J.A., Paterson, R.G., Takeda, M., Ohigashi, Y., Venkataraman, P., Lamb, R.A., and Pinto, L.H. (2003). Influenza B virus BM2 protein has ion channel activity that conducts protons across membranes. Dev Cell 5, 175-184.

Odagiri, T., Hong, J., and Ohara, Y. (1999). The BM2 protein of influenza $B$ virus is synthesized in the late phase of infection and incorporated into virions as a subviral component. J Gen Virol 80, 2573-2581.

Ohtsuka, K., and Hata, M. (2000). Molecular chaperone function of mammalian Hsp70 and Hsp40-a review. Int J Hyperthermia 16, 231-245.

Olsen, C.W., Kehren, J.C., Dybdahl-Sissoko, N.R., and Hinshaw, V.S. (1996). bcl-2 alters influenza virus yield, spread, and hemagglutinin glycosylation. J Virol 70, 663-666.

Pinto, L.H., Holsinger, L.J., and Lamb, R.A. (1992). Influenza virus M2 protein has ion channel activity. Cell 69, 517-528.

Pinto, L.H., and Lamb, R.A. (2006). The M2 proton channels of 
influenza A and B viruses. J Biol Chem 281, 8997-9000.

Schultz-Cherry, S., Dybdahl-Sissoko, N., Neumann, G., Kawaoka, Y., and Hinshaw, V.S. (2001). Influenza virus ns1 protein induces apoptosis in cultured cells. J Virol 75, 7875-7881.

Schultz-Cherry, S., and Hinshaw, V.S. (1996). Influenza virus neuraminidase activates latent transforming growth factor beta. J Virol 70, 8624-8629.

Shimbo, K., Brassard, D.L., Lamb, R.A., and Pinto, L.H. (1996). Ion selectivity and activation of the M2 ion channel of influenza virus. Biophys J 70, 1335-1346.

Sohn, S.Y., Kim, J.H., Baek, K.W., Ryu, W.S., and Ahn, B.Y. (2006). Turnover of hepatitis $B$ virus $X$ protein is facilitated by Hdj1, a human Hsp40/DnaJ protein. Biochem Biophys Res Commun 347, 764-768.

Srivastava, S.P., Kumar, K.U., and Kaufman, R.J. (1998). Phosphorylation of eukaryotic translation initiation factor 2 mediates apoptosis in response to activation of the double-stranded RNAdependent protein kinase. J Biol Chem 273, 2416-2423.

Sugrue, R.J., and Hay, A.J. (1991). Structural characteristics of the
M2 protein of influenza A viruses: evidence that it forms a tetrameric channel. Virology 180, 617-624.

Takeda, M., Pekosz, A., Shuck, K., Pinto, L.H., and Lamb, R.A. (2002). Influenza a virus M2 ion channel activity is essential for efficient replication in tissue culture. J Virol 76, 1391-1399.

Tan, S.L., Gale, M.J. Jr, and Katze, M.G. (1998). Double-stranded RNA-independent dimerization of interferon-induced protein kinase PKR and inhibition of dimerization by the cellular P58IPK inhibitor. Mol Cell Biol 18, 2431-2443.

Wurzer, W.J., Planz, O., Ehrhardt, C., Giner, M., Silberzahn, T., Pleschka, S., and Ludwig, S. (2003). Caspase 3 activation is essential for efficient influenza virus propagation. EMBO J 22, 2717-2728.

Yan, J., Zhu, J., Zhong, H., Lu, Q., Huang, C., and Ye, Q. (2003). BRCA1 interacts with FHL2 and enhances FHL2 transactivation function. FEBS Lett 553, 183-189.

Zamarin, D., García-Sastre, A., Xiao, X., Wang, R., and Palese, P. (2005). Influenza virus PB1-F2 protein induces cell death through mitochondrial ANT3 and VDAC1. PLoS Pathog 1, e4. 